\title{
Os Ichneumonidae (Hymenoptera) da Estação Ecológica da Universidade Federal de Minas Gerais, Belo Horizonte, com ênfase nas espécies de Pimplinae ${ }^{1}$
}

\author{
Alice Fumi Kumagai ${ }^{2}$
}

\begin{abstract}
Aвstract. The Ichneumonidae (Hymenoptera) of the Estação Ecológica of the Universidade Federal de Minas Gerais, Belo Horizonte, with emphasis on the Pimplinae species. In one annual cycle 83,712 insects were captured, of which 2,339 were Ichneumonidae, belonging to 17 subfamilies; they were collected by a Malaise trap placed in a montane semidecidual seasonal forest, inside the campus. Among the collected ichneumonids there were 13 genera and 30 species of Pimplinae, of which Pimpla croceiventris was the most frequent species. The species richness of Pimplinae was greater in the ecological station in Belo Horizonte, MG than in other localities studied.
\end{abstract}

Keywords. Hymenoptera; Ichneumonidae; Malaise trap; Pimplinae; species richness.

\section{INTRODUÇÃO}

Os icneumonídeos, em número de espécies, constituem uma das maiores famílias de insetos. A icneumofauna mundial é estimada em 60.000 espécies, das quais, aproximadamente, 17.000 ocorrem na Região Neotropical; destas, somente $10 \%$ é descrita (Townes 1969). São poucos os trabalhos de levantamento de Ichneumonidae e, na Região Neotropical, foram feitos por GAULD $(1991,1997)$ na Costa Rica; LANFRANCO (1974a,b), Jerez et al. (1977) e Porter (1979) com os icneumonídeos do Chile e PorTer (1975) com a fauna do noroeste argentino. No Brasil, um levantamento de Ichneumonidae foi feito por Kumagai \& GRAF (2000) na região de Curitiba, Paraná.

Pimplinae é uma subfamília moderadamente grande, com aproximadamente 60 gêneros descritos. Segundo GASTON \& GAULD (1993), algumas subfamílias e tribos são pouco representadas nos trópicos, outras mais ricas em espécies nas regiões temperadas e Pimplinae possui maior diversidade nos trópicos. Os Pimplinae são relativamente abundantes em coleções e em trabalhos de levantamentos, mas a sua identificação é dificultada pela grande variação cromática e falta de revisões taxonômicas. Os trabalhos de Townes \& TOwnes (1966), Townes (1969) e GAuld (1991), foram essenciais para o estudo dos gêneros e espécies de Pimplinae.

Os objetivos deste trabalho foram efetuar o levantamento da fauna de Ichneumonidae, com ênfase às espécies de
Pimplinae, da Estação Ecológica da Universidade Federal de Minas Gerais, e comparar os dados obtidos com os de trabalhos similares realizados em outras localidades.

\section{MATERIALEMÉTODOS}

A cidade de Belo Horizonte está localizada a $43^{\circ} 58^{\prime}$ W, $19^{\circ} 52^{\prime} \mathrm{S}$ e a uma altitude de $850 \mathrm{~m}$. As médias anuais de temperatura estão entre 20 e $22^{\circ} \mathrm{C}$ (isentos de geadas) e de precipitação de 1.500 a $1.750 \mathrm{~mm}$ (com três meses de seca: junho a agosto) (IBGE 1977).

Área de amostragem. A Estação Ecológica da Universidade Federal de Minas Gerais situa-se no campus da Pampulha. Possui 102 hectares de área e com a vegetação predominante resultante de recomposição paisagística, ocorrendo ainda matas de galeria, cerrado, mata secundária, "capineiras", eucaliptos (Eucalyptus sp.) (Myrtaceae) e brejos com taboas (Typha dominguensis Pers.) (Typhaceae) que ocupam parte do lago assoreado no lado oeste. Existem, hoje, capões isolados de vegetação arbórea circundados por gramíneas e espécies ornamentais, que constituem os remanescentes da cobertura vegetal nativa (ECodinÂMica 1991). Na classificação da vegetação, segundo Veloso \& Góes (1982), a área é de floresta estacional semidecidual montana. Uma armadilha de Malaise foi instalada no interior da mata secundária sub-perenifólia

1. Contribuição n 1314 do Departamento de Zoologia, Universidade Federal do Paraná. Parte da tese de doutorado, Departamento de Zoologia, UFPR.

2. Departamento de Zoologia, Universidade Federal de Minas Gerais. Caixa Postal 486, 31.270-901 Belo Horizonte-MG, Brasil.

Endereço eletrônico: acfk@icb.ufmg.br 
“regenerada" (Figs. 1, 2 ).

Armadilha utilizada. Utilizou-se uma armadilha de Malaise (modelo Townes 1972a) para a captura dos insetos. Foram obtidas amostragens semanais (53 amostras) durante um ciclo anual, no período de 13 de maio de 1991 a 18 de maio de 1992.

Identificação do material. Os gêneros de Pimplinae foram identificados com auxílio das publicações de Townes \& TownES (1966), Townes (1969) e GAULD (1991); no nível específico foram utilizados os trabalhos de GAuLd (1991) para os gêneros Acrotaphus, Zatypota e Neotheronia; PORTER (1970) para Pimpla [= Coccygomimus]; GRAF (1985) para Clistopyga e GraF \& Kumagai (1997) para Flacopimpla. A numeração das espécies não identificadas em nível específico segue a de KuMAGAI \& GraF (2000).

Análise dos dados. As espécies dominantes (em número) foram determinadas pelo método de KATO et al. 1952 (in LAROCA 1995 - programa “pg10-5.bas" BASIC) e os índices de ocorrência e dominância das espécies de Pimplinae foram obtidos pelo método proposto por Palma (1975) (in Abreu \& NogueIRA 1989).

Depósito do material. O material deste trabalho, foi depositado no Departamento de Zoologia do Instituto de Ciências Biológicas da Universidade Federal de Minas Gerais, em Belo Horizonte, Minas Gerais.

\section{RESULTADOS E DISCUSSÃO}

Foram capturados 83.712 insetos, sendo os Diptera,
Hymenoptera e Lepidoptera os mais freqüentes com 72,2\%, $8,4 \%$ e $6,8 \%$ do total, respectivamente. As ordens menos freqüentes foram: Ephemeroptera com um, Strepsiptera com quatro e Thysanoptera com dez indivíduos. Foram obtidos 501 Neuroptera em 36 das 53 amostras (67,9\%); as maiores freqüências foram de julho a setembro com a máxima semanal de 74 indivíduos (12 de agosto); a espécie mais abundante foi Chrysoperla externa (Hagen, 1861). A freqüência de Isoptera (414 indivíduos) esteve concentrada nos meses de setembro a início de janeiro. Os meses de outubro, novembro e dezembro foram os de maior captura de insetos $(13.365,11.709$ e 13.525), coincidindo com as mais altas médias de temperaturas máxima $\left(28,9^{\circ} \mathrm{C}\right)$ e mínima $\left(19,3^{\circ} \mathrm{C}\right)$ e as menores foram em julho e junho com 3.595 e 3.237 indivíduos, com temperaturas de $14,9^{\circ} \mathrm{C}$ (julho) e $15,8^{\circ} \mathrm{C}$ (junho) (Tabela I ). Na freqüência semanal, a variação foi de 4.641 (21 de outubro) a 558 insetos ( 22 de julho).

O total de insetos capturados em Belo Horizonte é semelhante ao de Telêmaco Borba e Fênix, no Paraná (MARINONI \& Dutra 1991), e maior que o de Curitiba, Paraná, nesta última localidade, uma mata de localização urbana (KUMAGAI \& GRAF 2000).

Os Ichneumonidae (2.339 exemplares) estiveram presentes em todas as 53 amostras. Das 17 subfamílias que ocorreram no local, Cryptinae foi a mais freqüente com 1.049 exemplares (44,8\%), seguida por Ichneumoninae com 370 exemplares (15,8\%), Pimplinae com 306 indivíduos (13,0\%), Campopleginae com $174(7,4 \%)$ e Orthocentrinae com 158 (6,7\%). As demais subfamílias coletadas estavam representadas por números menores de exemplares: Banchinae (68 indivíduos), Cremastinae (63), Ophioninae (45), Anomaloninae (27), Microleptinae (22), Mesochorinae (15), Labeninae(12), Tryphoninae (10), Ctenopelmatinae (10), Metopiinae (5), Tersilochinae (3) e

Tabela I. Total mensal das ordens dos insetos capturados com armadilha de Malaise na Estação Ecológica da Universidade Federal de Minas Gerais, em Belo Horizonte - MG, no período de maio de 1991 a maio de 1992.

\begin{tabular}{|c|c|c|c|c|c|c|c|c|c|c|c|c|c|c|c|c|}
\hline Mês & CI & Ep & $\mathrm{Od}$ & Or & Is & Ps & Th & $\mathrm{He}$ & Ho & $\mathrm{Ne}$ & Co & $\mathrm{St}$ & Le & $\mathrm{Di}$ & $\mathrm{Hy}$ & Total \\
\hline mai & 3 & & 1 & 7 & & 45 & & 50 & 112 & 2 & 19 & & 197 & 2.208 & 183 & 2.827 \\
\hline jun & 7 & & 1 & 16 & & 160 & 2 & 37 & 250 & 13 & 67 & 1 & 343 & 2.052 & 288 & 3.237 \\
\hline jul & 6 & & & 9 & & 166 & & 67 & 308 & 104 & 49 & 1 & 538 & 2.059 & 288 & 3.595 \\
\hline ago & 2 & 1 & 1 & 16 & & 219 & & 52 & 345 & 178 & 74 & & 867 & 1.879 & 316 & 3.950 \\
\hline set & 23 & & 8 & 26 & 74 & 200 & & 24 & 706 & 168 & 167 & & 673 & 3.111 & 945 & 6.125 \\
\hline out & 86 & & 1 & 40 & 13 & 95 & & 23 & 761 & 13 & 366 & 2 & 632 & 10.402 & 931 & 13.365 \\
\hline nov & 240 & & & 34 & 104 & 93 & & 36 & 479 & 2 & 549 & & 536 & 8.384 & 1.252 & 11.709 \\
\hline dez & 153 & & & 112 & 222 & 55 & 3 & 11 & 506 & 1 & 404 & & 475 & 10.545 & 1.38 & 13.525 \\
\hline jan & 113 & & & 60 & 1 & 26 & & 5 & 147 & 1 & 105 & & 176 & 4.335 & 368 & $5.33^{\circ}$ \\
\hline fev & 114 & & & 13 & & 13 & 1 & 3 & 173 & 3 & 46 & & 160 & 3.968 & 269 & 4.76 \\
\hline mar & 82 & & & 20 & & 45 & 4 & 3 & 193 & 4 & 60 & & 466 & 3.852 & 404 & 5.133 \\
\hline abr & 97 & & & 21 & & 111 & & 22 & 198 & 8 & 39 & & 396 & 2.404 & 502 & 3.798 \\
\hline mai & 97 & & 2 & 45 & & 102 & & 22 & 161 & 4 & 24 & & 269 & 5.293 & 329 & 6.34 \\
\hline Total & 1.023 & 1 & 14 & 419 & 414 & 1.330 & 10 & 355 & 4.339 & 501 & 1.969 & 4 & 5.728 & 60.492 & 7.113 & 83.71 \\
\hline
\end{tabular}

$\mathrm{Cl}$ = Collembola; Ep = Ephemeroptera; Od = Odonata; Or = Orthoptera; Is = Isoptera; Ps = Psocoptera; Th = Thysanoptera; He = Hemiptera; Ho $=$ Homoptera $; \mathrm{Ne}=$ Neuroptera Co $=$ Coleoptera $; \mathrm{St}=$ Strepsiptera $;$ Le $=$ Lepidoptera $;$ Di $=$ Diptera Hy $=$ Hymenoptera . 


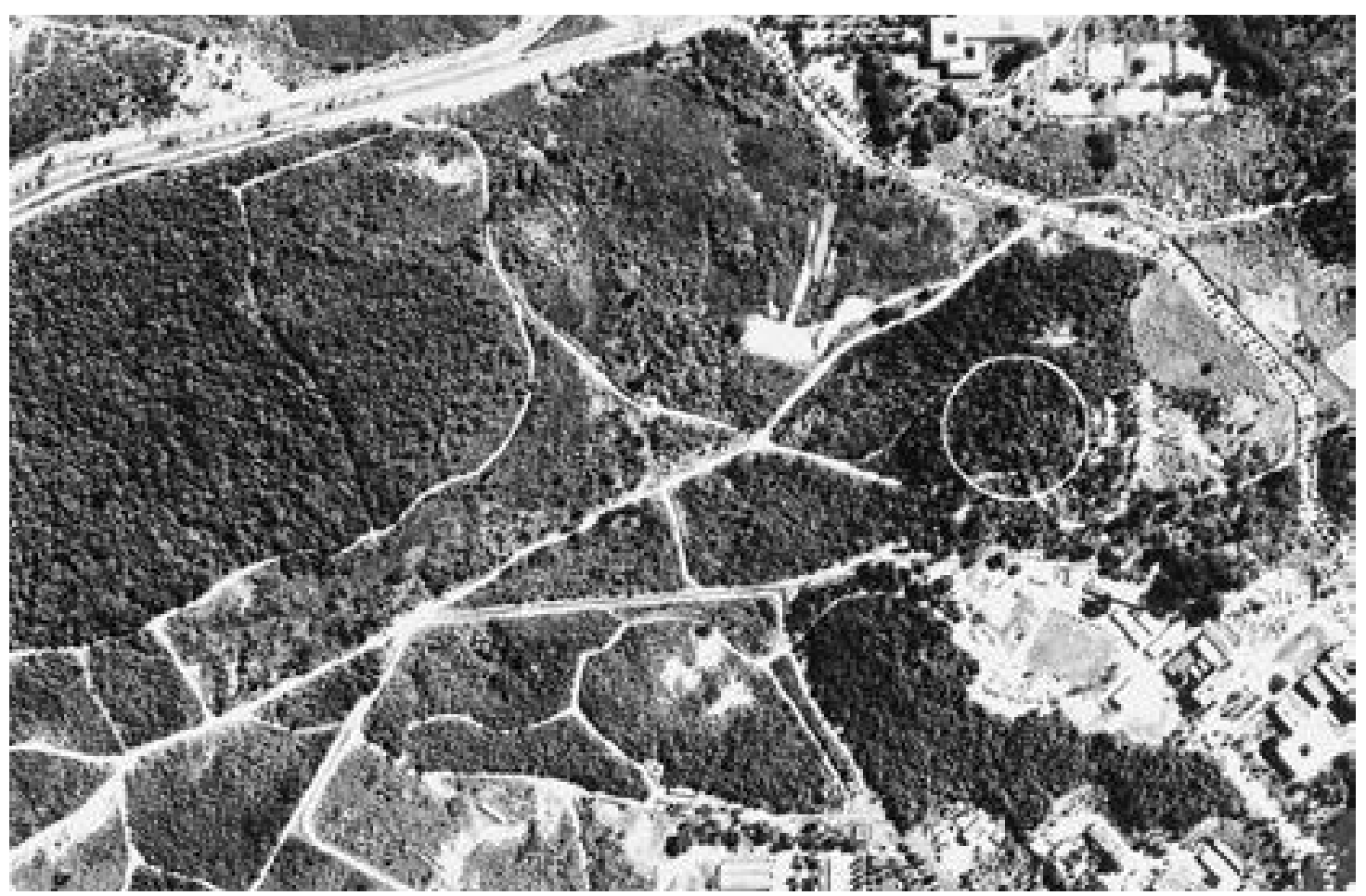

Fig. 1. Vista aérea de Belo Horizonte, Minas Gerais e local de amostragem (Estação Ecológica da Universidade Federal de Minas Gerais). (Fonte: Processamento de Dados do Município de Belo Horizonte - Prodabel; escala: 1/8000; 1994).

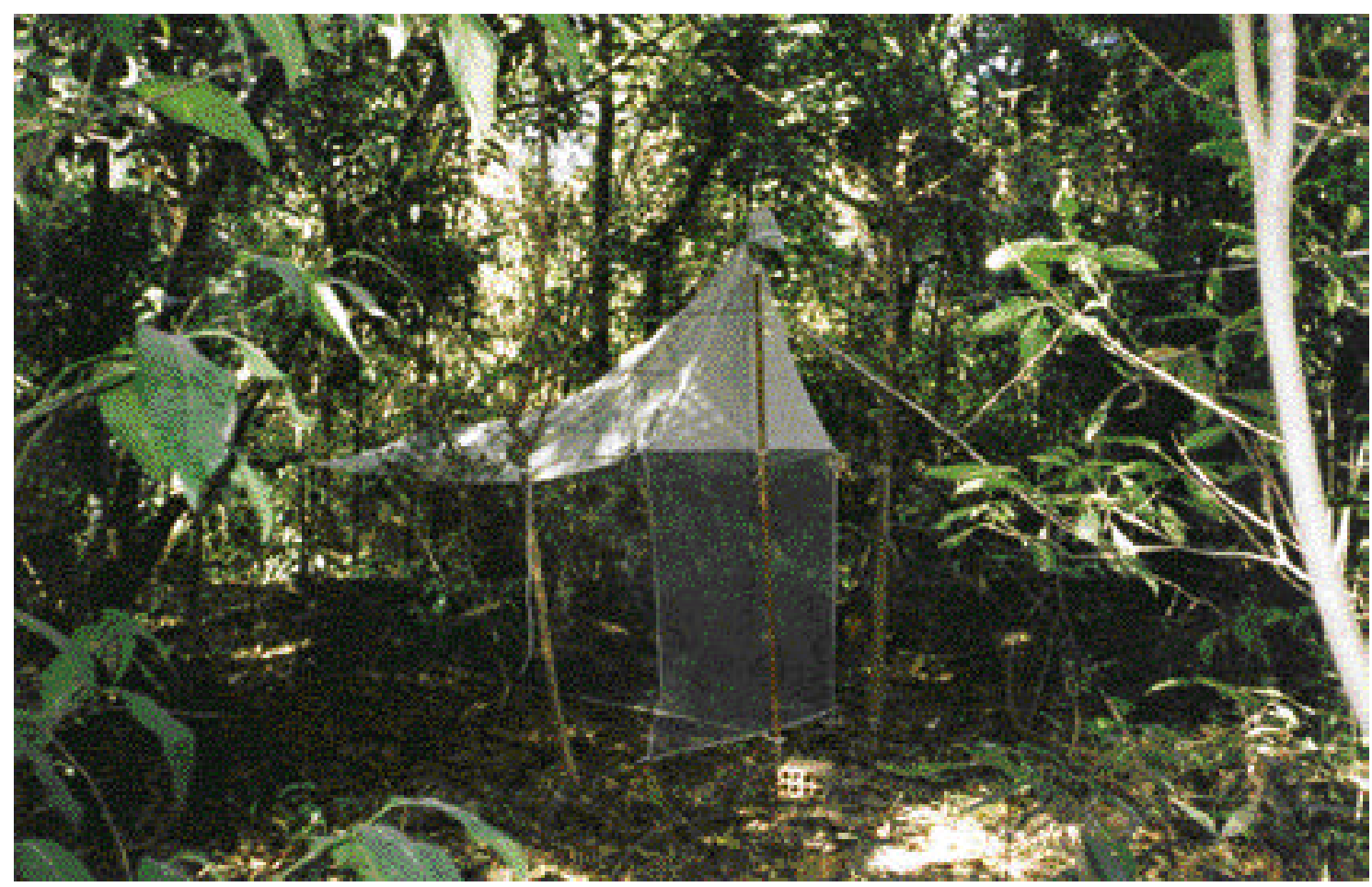

Fig. 2. Armadilha de Malaise (modelo Townes 1972) instalada no interior da mata da Estação Ecológica da Universidade Federal de Minas Gerais, em Belo Horizonte, Minas Gerais. 
Tabela II. Lista das espécies de Pimplinae da Estação Ecológica da Universidade Federal de Minas Gerais, em Belo Horizonte, Minas Gerais e a classificação de Palma (1975) para ocorrência e dominância.

\begin{tabular}{|c|c|c|}
\hline Espécies & $\mathrm{N}$ & $\begin{array}{c}\text { Classificação } \\
\text { DE PALMA } \\
(1975) \\
\end{array}$ \\
\hline \multicolumn{3}{|l|}{ PIMPLINAE } \\
\hline 01 Caliephialtes sp 5 & 1 & $\mathrm{R}$ \\
\hline 02 Anastelgis sp1 & 1 & $\mathrm{R}$ \\
\hline 03 Zonopimpla sp 1 & 1 & $\mathrm{R}$ \\
\hline 04 Zonopimpla sp 2 & 8 & $\mathrm{I}$ \\
\hline 05 Tromatobia sp 1 & 11 & $\mathrm{I}$ \\
\hline 06 Tromatobia sp 6 & 3 & $\mathrm{R}$ \\
\hline 07 Zaglyptus sp 2 & 1 & $\mathrm{R}$ \\
\hline 08 Clistopyga jakobi Graf, 1985 & 1 & $\mathrm{R}$ \\
\hline 09 Polysphincta sp 2 & 2 & $\mathrm{R}$ \\
\hline 10 Acrotaphus chedelae Gauld, 1991 & 8 & $\mathrm{I}$ \\
\hline 11 Acrotaphus fasciatus (Brullé, 1846) & 2 & $\mathrm{R}$ \\
\hline 12 Hymenoepimecis sp 1 & 9 & $\mathrm{I}$ \\
\hline 13 Hymenoepimecis sp 3 & 1 & $\mathrm{R}$ \\
\hline 14 Hymenoepimecis sp 4 & 1 & $\mathrm{R}$ \\
\hline 15 Hymenoepimecis sp 5 & 1 & $\mathrm{R}$ \\
\hline 16 Flacopimpla sulina Graf \& Kumagai, 1997 & 17 & $\mathrm{I}$ \\
\hline 17 Zatypota alborhombarta (Davis, 1895) & 3 & $\mathrm{R}$ \\
\hline 18 Neotheronia lineata (Fabricius, 1804) & 37 & $\mathrm{I}$ \\
\hline 19 Neotheronia tacubaya (Cresson, 1874) & 1 & $\mathrm{R}$ \\
\hline 20 Neotheronia lloydi Gauld, 1991 & 24 & $\mathrm{I}$ \\
\hline 21 Neotheronia montezuma (Cresson, 1874) & 2 & $\mathrm{R}$ \\
\hline 22 Neotheronia donovani Gauld, 1991 & 1 & $\mathrm{R}$ \\
\hline 23Neotheronia alfaroae Gauld, 1991 & 1 & $\mathrm{R}$ \\
\hline 24 Neotheronia chiriquensis (Cameron, 1886) & 19 & $\mathrm{I}$ \\
\hline 25 Neotheronia aff. cherfasi & 8 & $\mathrm{I}$ \\
\hline 26 Neotheronia tolteca (Cresson, 1874) & 4 & $\mathrm{R}$ \\
\hline 27 Pimpla sumichrasti Cresson, 1874 & 1 & $\mathrm{R}$ \\
\hline 28 Pimpla azteca Cresson, 1874 & 5 & $\mathrm{R}$ \\
\hline 29 Pimpla croceiventris (Cresson, 1868) & 77 & $\mathrm{C}$ \\
\hline 30 Pimpla golbachi (Porter, 1970) & 55 & $\mathrm{I}$ \\
\hline
\end{tabular}

Total $=306$

$\mathrm{N}$ = frequiência de indivíduos; Classificação de Palma (1975): $\mathrm{R}=$ rara; $\mathrm{I}=$ intermediária e $\mathrm{C}=$ comum.

Diplazontinae (2). A Fig. 3 representa a frequiência mensal de Ichneumonidae e a precipitação pluviométrica no período de coleta. Os maiores picos populacionais ocorreram entre setembro (344 indivíduos) e novembro (373 exemplares). A maior captura mensal foi em novembro com a média semanal de 93,2 indivíduos e a menor foi em junho, com 77 indivíduos e a média semanal de 19,2. Em janeiro e fevereiro as freqüências foram mais baixas ( 84 e 89 indivíduos) e estão relacionadas às duas maiores precipitações semanais $(204,2$ e $166,5 \mathrm{~mm}$ ) ou aos 22 dias de chuva em janeiro e que também influenciaram na captura de fevereiro. Nos meses seguintes, houve um aumento gradativo até abril (182 indivíduos). No período da coleta a temperatura máxima média semanal variou de $29,8^{\circ} \mathrm{C}$ a $21,5^{\circ} \mathrm{C}$; a temperatura mínima média semanal esteve entre $20,4^{\circ} \mathrm{C}(6 \mathrm{de}$ abril) e $13,4^{\circ} \mathrm{C}$ ( 26 de agosto). Estes intervalos de temperatura são propícios à atividade de vôo dos icneumonídeos, que necessitam também de água, diariamente, na forma de chuva ou orvalho sobre as folhas (Townes 1958, 1972b). O período seco, entre o final de maio e o início de setembro (14 semanas) foi de baixa frequiência de indivíduos (média de 100 indivíduos/ mês) e com o início das chuvas houve um aumento na captura (mais de 300 indivíduos/mês) (Fig. 3 ). Na comparação com as capturas feitas em Curitiba (KumAGAI \& GRAF 2000), as menores ocorrências foram verificadas no inverno e estão relacionadas às baixas temperaturas do mês de junho $\left(3,6^{\circ} \mathrm{Ce} 6,8^{\circ} \mathrm{C}\right)$.

Os 306 exemplares de Pimplinae coletados representam 13,0\% do total de Ichneumonidae, distribuídos em 13 gêneros e 30 espécies, sendo que 12 espécies ocorreram com somente um exemplar (Tabela II ). Os gêneros Neotheronia e Pimpla foram os mais numerosos em indivíduos $(31,6 \%$ e 45,0\% do total de Pimplinae) e também os mais ricos em espécies, com nove e sete, respectivamente. Resultados semelhantes também foram obtidos em localidades da Costa Rica (GAULD 1991) e do Peru (CARRASCo 1972).

Pelo método de Kato et al. (1952), conforme Laroca (1995), as espécies Pimpla croceiventris, P. golbachi, Neotheronia lineata, Neotheronia lloydi, Neotheronia chiriquensis e Flacopimpla sulina são consideradas dominantes (em número de indivíduos), com 77, 55, 37, 24, 19 e 17 exemplares, respectivamente, e representam $74,8 \%$ dos pimplíneos capturados. A distribuição sazonal destas espécies é apresentada na Tabela III.

Na classificação de Palma (1975) (in ABreu \& Nogueira 1989), para a ocorrência e dominância das 30 espécies, Pimpla

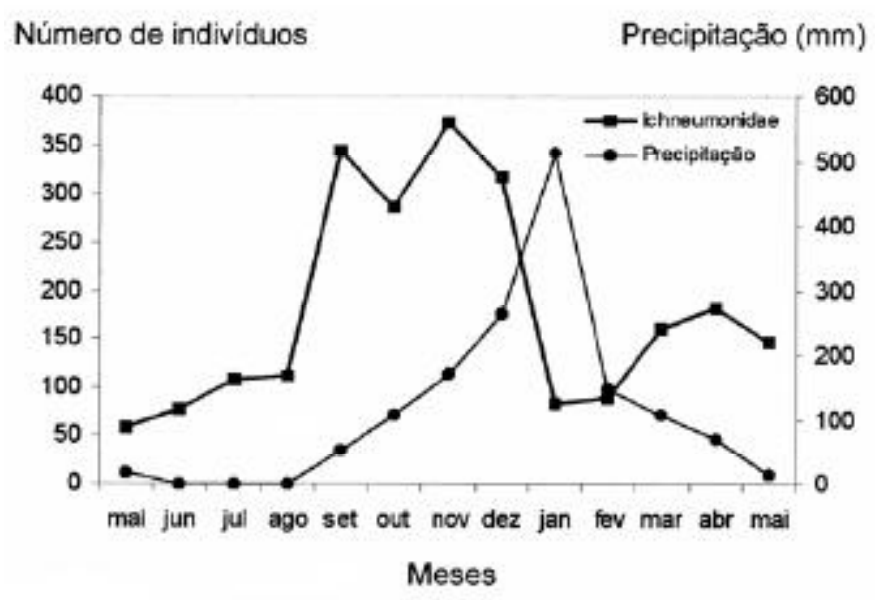

Fig. 3. Freqüencia mensal de indivíduos de Ichneumonidae e precipitação pluviométrica na Estação Ecológica da UFMG no período de maio de 1991 a maio de 1992. 
Tabela III. Distribuição sazonal e freqüência das espécies dominantes (classificação de KATo et al. 1952) de Pimplinae da Estação Ecológica da Universidade Federal de Minas Gerais, em Belo Horizonte, Minas Gerais.

\begin{tabular}{l|c|c|c|c|c|c|c|c|c|c|c|c|c}
\hline \multicolumn{1}{c|}{ Espécies } & jan & fev & mar & abr & mai & jun & jul & ago & set & out & nov & dez & total \\
\hline Pimpla croceiventris & 6 & 4 & 11 & 11 & 17 & 4 & 1 & 2 & 2 & 1 & 3 & 15 & 77 \\
Pimpla golbachi & - & - & 1 & - & 1 & 2 & 2 & 10 & 19 & 10 & 7 & 3 & 55 \\
Neotheronia lineata & 2 & 1 & 3 & 3 & 2 & 3 & 1 & - & 2 & 6 & 7 & 7 & 37 \\
Neotheronia lloydi & 2 & - & 2 & 1 & 7 & - & 4 & - & 2 & 2 & 1 & 3 & 24 \\
Neotheronia chiriquensis & - & 1 & - & - & 4 & - & 1 & - & 7 & 2 & 2 & 2 & 19 \\
Flacopimpla sulina & 1 & - & - & - & - & - & - & 1 & - & 1 & 9 & 5 & 17 \\
\hline
\end{tabular}

croceiventris é considerada comum, dez são intermediárias (Pimpla golbachi, Neotheronia lineata, Neotheronia lloydi, Neotheronia chiriquensis, Flacopimpla sulina, Tromatobia sp. 1, Hymenoepimecis sp. 1, Zonopimpla sp. 2; Acrotaphus chedelae e Neotheronia aff. cherfasi) e as restantes são raras (Tabela II). Pimpla golbachi foi a segunda espécie mais freqüente mas não foi considerada como espécie comum devido à sua freqüência concentrada no segundo semestre do ano.

No catálogo dos Ichneumonidae neotropicais (TownEs \& Townes 1966) estão listados 24 gêneros de Pimplinae (= Ephialtinae); para a fauna da Costa Rica (GAULD 1991 e GASTON \& GAULD 1993) são apresentados 27 gêneros e aproximadamente 160 espécies; no Chile, PORTER (1979) registrou a ocorrência de 11 gêneros na Província de Tarapacá e áreas adjacentes; LANFRANCO (1974a) coletou apenas o gênero Pimpla no Parque "Vicente Perez Rosales"; os gêneros Tromatobia e Pimpla [Tromatobia sponsa (Haliday 1836) e Pimpla fuscipes Brullé, 1846] foram coletados na região de Magallanes (LanfRANCo, 1974b) e também no Bosque de Quintero (JEREZ et al. 1977); na província biogeográfica do Monte, no noroeste argentino, PORTER (1975) obteve sete gêneros e dez espécies. Comparando com os dados acima, podemos concluir que a fauna de Ichneumonidae - Pimplinae da Estação Ecológica da Universidade Federal de Minas Gerais (13 gêneros e 30 espécies) é mais rica que todas as localidades acima citadas, exceto Costa Rica, onde o esforço de coleta foi muitas vezes maior que o realizado neste trabalho.

\section{REFERÊNCIAS}

Abreu, P. C. O. V. \& C. R. Nogueira, 1989. Spatial distribution of Siphonophora species at Rio de Janeiro coast, Brazil. Ciência e Cultura 41(9):897-902.

Carrasco-Z., F. 1972. Catalogo de la familia Ichneumonidae peruanos. Revista Peruana de Entomologia 15(2):324-332.

ECODINÂMICA. 1991. Estudo ambiental: subsídio à construção das unidades de Odontologia e Farmácia no Campus da Pampulha. $52 \mathrm{p}$.

Gaston, K. J. \& I. D. Gauld. 1993. How many species of Pimplines (Hymenoptera: Ichneumonidae) are there in Costa Rica? Journal of Tropical Ecology 9:491-499.

Gauld, I. 1991. The Ichneumonidae of Costa Rica, 1. Memoirs of the American Entomological Institute 47:1-589.

Gauld, I. 1997. The Ichneumonidae of Costa Rica, 2. Memoirs of the American Entomological Institute 57:1-485.

GraF, V. 1985. Ichneumofauna do Sudeste e Sul do Brasil. VI. Nova espécie de Clistopyga (Ephialtinae, Hymenoptera). Revista Brasileira Entomologia 29(2):349-350.

GraF, V. \& A. F. Kumagai. 1997. A ocorrência de Flacopimpla Gauld no Brasil (Hymenoptera, Ichneumonidae, Pimplinae). Revista Brasileira de Zoologia 14(4):773-777.

Instituto Brasileiro de Geografia e Estatística. 1977. Geografia do Brasil. Região Sudeste. Fundação Instituto Brasileiro de Geografia e Estatística, $667 \mathrm{p}$.

Jerez, V.; D. Lanfranco, \& B. Andrade. 1977. Aspectos ecologicos de los icneumonidos del Bosque de Quintero. Anales del Museo de Historia Natural de Valparaiso 10:161-168.

Kato, M.; T. Matsuta, \& Z. Yamashita. 1952. Associative ecology of insects found in the paddy field cultivated by various planting forms. Science Reports Tohoku University, IV (Biology). 19:291-301.

Kumagai, A. F. \& V. Graf. 2000. Ichneumonidae (Hymenoptera) de áreas urbana e rural de Curitiba, Paraná, Brasil. Acta Biológica Paranaense 28:153-168.

LANFrANCO, D. 1974a. Ichneumonidos (Hymenoptera - Ichneumonidae) del Parque Nacional "Vicente Perez Rosales". Anales del Museo de Historia Natural de Valparaiso 7:261-267.

LANFRANCO, D. 1974b. Contribución al conocimiento de la ichneumofauna de la region de Magallanes (Hymenoptera - Ichneumonidae). Anales del Instituto de la Patagonia 5(1-2):199-208.

Laroca, S. 1995. Ecologia - Princípios e métodos. Petrópolis, Ed. Vozes, $197 \mathrm{p}$.

Marinoni, R. C. \& R. R. C. Dutra. 1991 [1993]. Levantamento da fauna entomológica do Estado do Paraná. I. Introdução. Situação climática e florística dos oito pontos de coleta. Dados faunísticos de agosto de 1986 a junho de 1987 . Revista Brasileira de Zoologia 8(1/2/3/ 4):31-73.

PaLma, S. 1975. Contribución al estudio de los sifonoforos encontrados frente a la costa de Valparaiso. Aspectos ecologicos. In: II Simposio Latinoamericano sobre Oceanografia Biologica, Univ. d'Orient, Venezuela, 2:119-133.

Porter, C. 1970. A revision of the South American species of Coccygomimus (Hymenoptera, Ichneumonidae). Studia Entomologica 13(1-4):1-192.

PorTer, C. 1975. Relaciones zoogeograficas y origen de la fauna de Ichneumonidae (Hymenoptera) en la provincia biogeografica del Monte del noroeste argentino. Acta Zoologica Lilloana 31(15): 175-252.

PORTER, C. 1979. Ichneumonidae de Tarapacá. I. Subfamilia Ephialtinae (Hymenoptera). Idesia 5:157-187.

Townes, H. 1958. Some biological characteristics of the Ichneumonidae (Hymenoptera) in relation to biological control. Journal of 
Economic Entomology 51(5):650-652.

Townes, H. 1969. Genera of Ichneumonidae (Part 1). Memoirs of the American Entomological Institute 11:1-300.

Townes, H. 1972a. A light-weight Malaise trap. Entomological News 83(9):239-247.

Townes, H. 1972b. Ichneumonidae as biological control agents. Proceedings Tall Timbers Conference on Ecological Animal
Control by Habitat Management 3:235-248.

Townes, H. \& M. Townes. 1966. A catalog and reclassification of the Neotropic Ichneumonidae. Memoirs of the American Entomological Institute 8:1-367.

Veloso, P. H. \& L. Góes Filho. 1982. Fitogeografia brasileira. Classificação fisionômica-ecológica da vegetação neotropical. Boletim Técnico Projeto RADAMBRASIL, Série Vegetação, 85 p. 